\title{
Impact of chirality on phase diagrams and conversely relative quantification of chirality by using the binary phase diagram of enantiomers
}

\author{
G. Coquerel \\ Unité de Croissance Cristalline et de Modélisation Moléculaire, UPRES EA 2659, \\ IRCOF, Université de Rouen, 76821 Mont-Saint-Aignan Cedex, France
}

\begin{abstract}
The heterogeneous equilibria between two enantiomers can be depicted in a unary system or a binary system according to the possibility of interconvertion ( $\mathrm{n}=2$ if no racemization occurs). In case of a binary system, the symmetry between the chemical potentials introduces a modification in the Gibbs phase rule but the resulting GibbsScott phase rule is only applicable if the number of symmetrical phases differs from zero i.e. no racemic monophasic domain exists. At a given temperature a quantitative determination of the degrees of asymmetry of a molecule is proposed by using the stable (or metastable) binary heterogeneous equilibria with its antipode. For monophasic domains, this evaluation is based on the width in composition and on how far from the racemic mixture the boundaries are. For biphasic domains the evaluation corresponds to the algebraic departure in composition of the two co-existing phases.
\end{abstract}

\section{INTRODUCTION}

Study of molecular asymmetry originates from Pasteur's pioneer work in 1848 [1]. Since then, a huge amount of works have been devoted to the development of chirality in physics, chemistry, biochemistry, microbiology, catalysis, organic synthesis etc. Nevertheless, comparatively few articles can be found on the incidence of molecular asymmetry on thermodynamics [2 to 5]. The aim of this paper is to deal with that question and in return to propose a new way to tackle the old problem of chirality quantification by using heterogeneous equilibria. In order to simplify the notation, the enantiomers will be labelled hereafter as R and S whatever the nature of their asymmetry (e.g. RSSS and SRRR or axial asymmetry Ra and $\mathrm{Sa}$ ).

\section{NATURE OF THE R-S SYSTEM}

Symmetrical components, such as enantiomers, introduce an additional relation between the chemical potentials (weak forces such as those prevailing in $\beta$ decay are not considered in this paper). Therefore, whatever the phase $(\alpha)$ :

$$
\mu_{R}^{\alpha}=\mu_{S}^{\alpha}
$$

If the two enantiomers can interconvert, the $\mathrm{R} \leftrightarrow \mathrm{S}$ equilibrium is associated with no heat transfer $(\Delta H=0)$ and thus, whatever the temperature, the equilibrium constant is $\mathrm{K}=1(\forall \mathrm{T}$ the two antipodes have exactly the same $\mathrm{Cp}$ ). Consequently, the equilibrium is entropy driven only as depicted by the following formulae.

$$
\Delta G=-R T\left(x_{R} \ln x_{R}+x_{S} \ln x_{S}\right)
$$

If the enantiomers cannot interconvert then the heterogeneous equilibria can be described within the framework of a binary system but the additional relation between the chemical potentials (1) leads to a new version of the Gibbs phase rule (Gibbs-Scott phase rule) which takes this symmetry into account [6].

$$
v=\frac{n_{2}}{2}+n_{1}+2-\frac{\varphi_{2}}{2}-\varphi_{1}
$$

$\mathrm{n}_{1}$ and $\varphi_{1}$ stand respectively for the number of independent components and the number of phases which are not symmetrical.

$\mathrm{n}_{2}$ and $\varphi_{2}$ stand respectively for the number of independent components and the number of phases which are symmetrical (even figures). 
The symmetry appears also in the binary phase diagram as a vertical mirror parallel to the axis (axes) of the physical variable(s) ( $\mathrm{T}$ and/or $\mathrm{P}$ ) and orthogonal to the composition segment at $\mathrm{x}_{\text {mass }}=\mathrm{X}_{\mathrm{mol}}=0.5$

It is important to note that the Gibbs-Scott relation is applicable only if $\varphi_{2} \geq 2$; thus when symmetrical phases exist. Figures $1 \mathrm{a}, 1 \mathrm{~b}$ and $1 \mathrm{c}$ give an illustration of phases diagrams containing those symmetrical phases which upon reversible transitions lead to 4 phase invariants. Figures $2 a, 2 b$ and $2 c$ depict similar situations with partial solid solutions. By contrast, figures $3 a, 3 b$ and $3 c$ show that, at a given temperature, the presence of the racemic compound imposes $\varphi_{2}=0$.

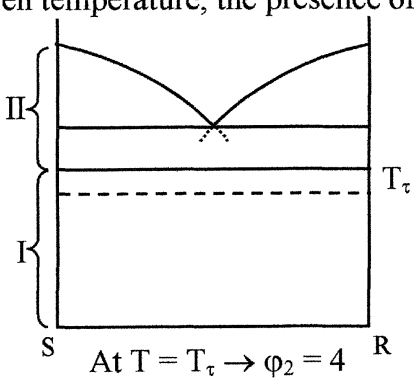

1a)

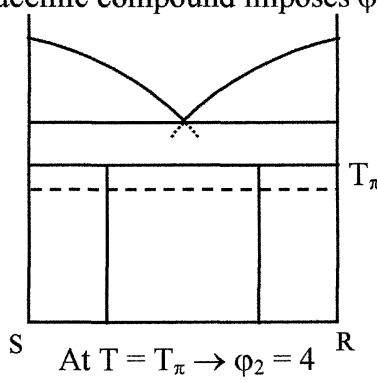

1b)

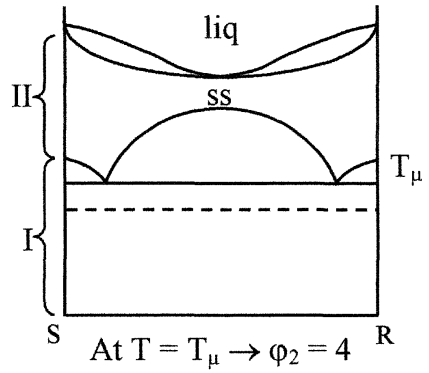

1c)

Figure 1: Various 4 phase invariants in binary phase diagrams between enantiomers (no solid solution) Tamman plot represented with dashed lines

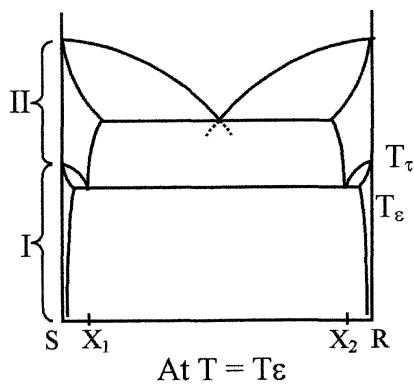

for $\mathrm{X} \in\left[\mathrm{X}_{1} ; \mathrm{X}_{2}\right] \rightarrow \varphi_{2}=4$

2a)

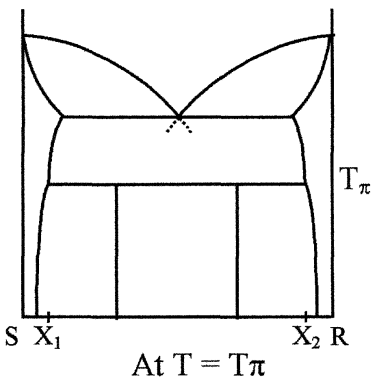

for $X \in\left[X_{1} ; X_{2}\right] \rightarrow \varphi_{2}=4$

$2 b)$

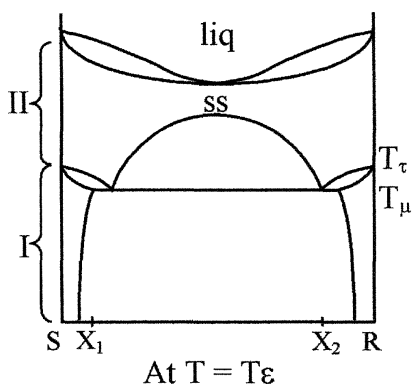

for $\mathrm{X} \in\left[\mathrm{X}_{1} ; \mathrm{X}_{2}\right] \rightarrow \varphi_{2}=4$

Figure 2: Various 4 phase invariants in binary phase diagrams between enantiomers (with partial solid solutions)

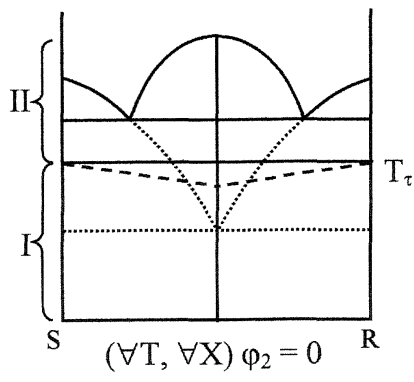

3a)

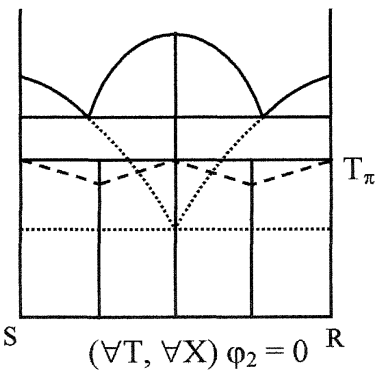

$3 b)$

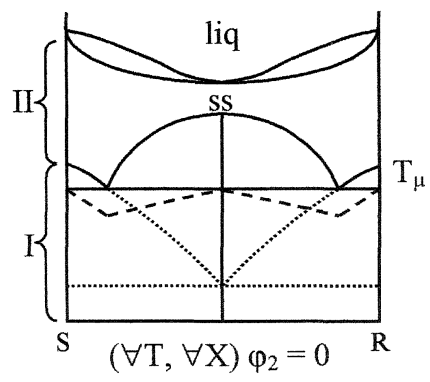

3c)

Figure 3: No 4 phase invariants nor co-existing symmetrical phases because of the presence of a racemic compound . Tamman plot represented with dashed lines

Up to now, literature reveals no experimental confirmation of any 4 phase invariant in a binary system of antipodes (at constant pressure). Several reasons can justify this lack of experimental evidence. The proportions of conglomerate and solid solution are poor so the existence of two conglomerates (fig. $1 \mathrm{a}$ and $2 \mathrm{a}$ ) or mixed crystals $(\forall \mathrm{x} \in[0 ; 1]$ ) and conglomerate (fig. 1c and $2 \mathrm{c}$ ) connected with two 
enantiotropic varieties of the components are likely to be of minor occurrences. The so-called 'abnormal' racemic compounds (whose stoichiometries differ from (1-1)) are even more exceptional [7]. Moreover, the solid-solid transitions at the solid state are associated with weak thermal effects and have usually a poor kinetics; these additional factors increase the difficulty to spot one of these low probability cases. Therefore, one of the easiest ways to give an experimental confirmation of the Gibbs-Scott phase rule is likely to be depicted on figure 4 where two conglomerates (one solvated and the other non solvated) coexist in a large domain of temperature and composition and a five phase invariant should be detected at the temperature of the binary peritectic transition (non congruent fusion of the solvates).

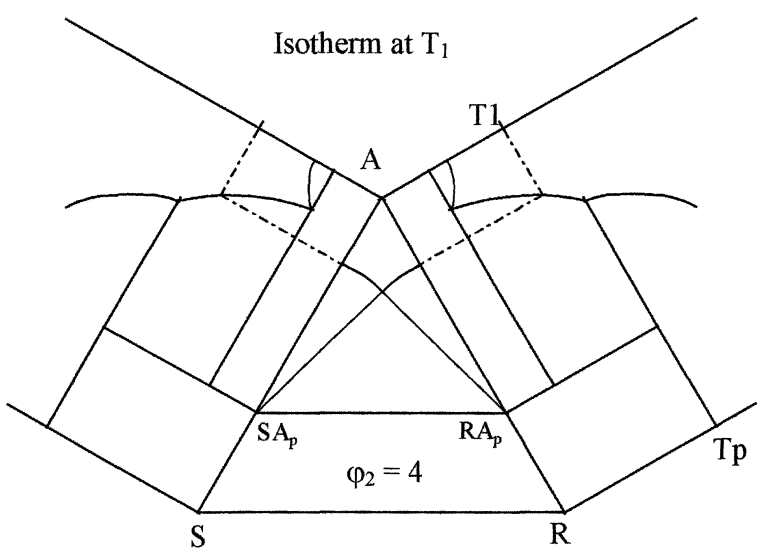

4 a

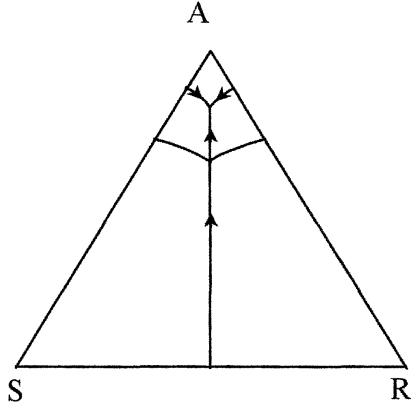

$4 b$

Figure 4: a) Isothermal section in the ternary system A-R-S which exhibits a four phase domain $\left(R, R A_{p}, S, S A_{p}\right)$;

b) Monovariant curves of the A-R-S system, at Tp : 5 phases should be in equilibrium : $\varphi_{1}=1$ (a racemic invariant saturated solution), $\varphi_{2}=4$ (two couples of symmetrical phases : $R, S, \mathrm{RA}_{\mathrm{p}}, \mathrm{SA}_{\mathrm{p}}$ )

\section{QUANTIFICATION OF CHIRALITY BY USING BINARY PHASE DIAGRAM}

Most of the time, the chirality is used as a qualitative concept (i.e. to the question : is the object chiral ? the answers are yes or no; $\chi=1$ or 0 ). Nevertheless, several attempts to quantify this notion have proved to be a difficult task [8]. Usually, the authors try to measure the difference between the two antipodes or sometimes between one enantiomer and an achiral reference [9]. In the author's opinion, any particular quantification of chirality has a restricted area of applicability (e.g. biological effects, chemistry : retention time in chiral chromatography, physics : magnitude of absolute rotatory power, intensity of the second harmonic generation, etc.). Moreover, in a given context, several functions designed to quantify the chirality can be arbitrarily defined. The quantification proposed here represents a first attempt to evaluate the asymmetry by using the binary phase diagrams of the enantiomers.

By definition, the enantiomeric excess (e.e. hereafter) represents the excess of an enantiomer with regards to the racemic mixture (the equal number of $\mathrm{R}$ and $\mathrm{S}$ molecules). Therefore, e.e. equals $-1,0$ and +1 for pure $\mathrm{S}$, racemic mixture and pure $\mathrm{R}$ respectively.

$$
\text { e.e. }=\frac{R-S}{R+S}
$$

In the binary system of enantiomers the degree of asymmetry is defined in monophasic domain by $\chi_{1}$ and in biphasic domain by $\chi_{2}$ as follows $\left(\chi_{1}\right.$ and $\left.\chi_{2} \in[0 ; 1]\right)$ :

$$
\chi_{1}=\left|\frac{e . e . l 1+e . e . l 2}{2}\right| \text { and } \chi_{2}=\left|\frac{e . e . l 1-e . e . l 2}{2}\right|
$$


e.e.11 and e.e.12 represent at a given temperature the limit on the left hand side and on the right hand side of the domain. For a monophasic domain restricted to a vertical line (e.g. the stoichiometric racemic compound or the pure enantiomer without solid solution) e.e. $11=$ e.e. 12 .

Figures $5 \mathrm{a}$ and $5 \mathrm{~b}$ illustrate various degrees of asymmetry at different temperatures and for different ranges in composition. These evaluations correspond to stable or metastable equilibria but unstable states could have led to an evaluation as well.
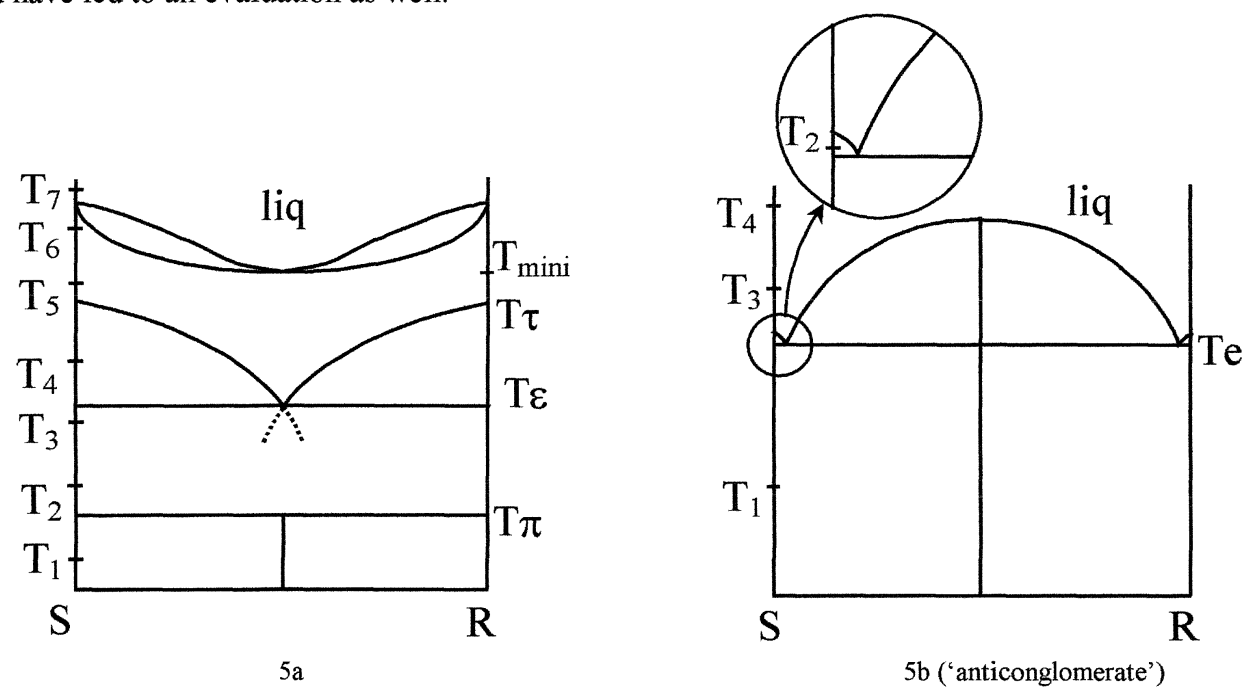

Figure 5: Quantification of chirality : Isothermal scans of the binary system from left to right

a) at $T_{1}: \chi_{1}=1 ; \chi_{2}=0.5 ; \chi_{1}=0 ; \chi_{2}=0.5 ; \chi_{1}=1$

at $\left.\mathrm{T}_{2}: \chi_{1}=1 ; \forall \mathrm{X} \in\right] 0 ; 1\left[\chi_{2}=1 ; \chi_{1}=1\right.$

at $\mathrm{T}_{3}$ : System in thermodynamic equilibrium same values as those at $T_{2}$

System in metastable equilibrium (dotted lines) between $\mathrm{S}$ and the corresponding supercooled molten state :

$\chi_{1}=1 ; \chi_{2}=0.54$.

at $\mathrm{T}_{4} \chi_{1}=1 ; \chi_{2}=0.39 ; \chi_{1}=0 ; \chi_{2}=0.39 ; \chi_{1}=1$

at $\mathrm{T}_{5} \forall \mathrm{X} \in[0 ; 1] \chi_{1}=0$

at $\mathrm{T}_{6} \chi_{1}=0.96 ; \chi_{2}=0.16 ; \chi_{1}=0 ; \chi_{2}=0.16 ; \chi_{1}=0.96$

at $\mathrm{T}_{7} \forall \mathrm{X} \in[0 ; 1] \chi_{1}=0$

b) at $T_{1}: \chi_{1}=1 ; \chi_{2}=0.5 ; \chi_{1}=0 ; \chi_{2}=0.5 ; \chi_{1}=1$

at $\mathrm{T}_{2} \chi_{1}=1 ; \chi_{2}=0.02 ; \chi_{1}=0.95 ; \chi_{2}=0.47 ; \chi_{1}=0 \chi_{2}=0.47 ; \chi_{1}=0.95 ; \chi_{2}=0.02 ; \chi_{1}=1$

at $\mathrm{T}_{3} \chi_{1}=0.9 ; \chi_{2}=0.4 ; \chi_{1}=0 ; \chi_{2}=0.4 ; \chi_{1}=0.9$

at $\mathrm{T}_{4} \forall \mathrm{X} \in[0 ; 1] \chi_{1}=0$

In a monophasic domain and for $\left(\mathrm{T}, \mathrm{X} \in \mathrm{XX}_{11} ; \mathrm{X}_{12}[), \chi_{1}\right.$ characterizes how far the molecules of the same handedness can flock together in a single phase. For $\chi_{1}$ values approaching 1 it means that the domain is narrow in composition and close to the pure enantiomer; the asymmetry of a given molecule is highly recognized by molecules of the same and opposite handedness. By opposition, for $\chi_{1}$ values close to 0 , a poor homochiral recognition is observed. From the examples displayed on figures $5 \mathrm{a}$ and $5 \mathrm{~b}$ it appears that any monophasic domain expanding symmetrically on both sides of $\mathrm{X}=0.5$ corresponds to $\chi_{1}=0$. From figure $5 \mathrm{a}$, it appears that a pure enantiomer can be associated to $\chi_{1}$ values ranging from 1 to 0 . For instance $: \chi_{1}=1$ for $\left.\mathrm{T} \in\right] \mathrm{T} \pi ; \mathrm{T} \varepsilon\left[; \chi_{1}=0.5\right.$ for $\mathrm{T}<\mathrm{T} \pi ;$ or even $\chi_{1}=0$ for $\left.\mathrm{T} \in\right] \mathrm{T} \tau$; Tmini[ because its crystal lattice can cope with both enantiomers in the whole range of composition (complete solid solution).

Inside a biphasic domain and for $(\mathrm{T}, \mathrm{X} \in] \mathrm{X}_{11} ; \mathrm{X}_{12}[), \chi_{2}$ characterizes how stereoselective the distribution of the enantiomers between the two phases is. The farther apart $X_{11}$ and $X_{12}$, the larger $\chi_{2}$; ideally each enantiomer is selectively surrounded by molecules of the same handedness only : i.e. the conglomerate. In case of binary system of an 'anti-conglomerate' type (racemic compound with a thermal 
stability much greater than that of the enantiomer, see figure $5 b$ ), the liquid phase -with a e.e. which can be in practice lower than 0.01 - is in equilibrium with the racemic compound at $\mathrm{T} 2$ slightly above Te. For $\mathrm{Xe}<\mathrm{X}<0.5$, the distribution of the chiral molecules in the two phases is highly contrasted. The situation with $\mathrm{Xe}<0.01$ is often overlooked although it can be beneficial to ensure an enantiomeric purification with hardly any loss of pure enantiomer.

\section{CONCLUSION}

The strict identity of the chemical potentials of enantiomeric components results in a mirror symmetry in the phase diagram through the composition $X=0.5$. Because of this symmetry, the Gibbs phase rule must be modified accordingly. The adapted relation (Gibbs-Scott relation) can only be applied if the number of symmetrical phases is different from zero.

The binary phase diagram between a couple of antipodes provides a simple and 'natural' means to quantify the degrees of asymmetry of chiral solutes in a well defined context : nature of the system, temperature, range in composition. With the benefit of hindsight, the diversity in the degrees of asymmetry found in this simple context gives evidence that the quantification of chirality is a difficult problem to encompass in its global aspect.

\section{Acknowledgements}

Thanks are due to Dr M-N Petit for help in the illustrations

\section{References}

1. W.J. Lough and I.W. Wainer, Chirality in Natural and Applied Science, Blackwell Science Ltd, 2002, Oxford

2. H. A. Oonk, Phase rule theory, Elsevier, 1981

3. G. Coquerel, Enantiomer, 2000, 5, 481-498

4. M. Farina, J. Chem. Soc. Chem. Commun., 1987, 1121-2

5. J. S. Chickos \& D. G. Hesse, Struct. Chem., 1991, Vol. 2, 33-40

6. R.L. Scott, J. Chem. Soc. Faraday trans. II, 1977, (3) p356-60

7. H.E. Gallis, P.J. van Ekeren, J.C. van Miltenburg, H.A.J. Oonk, Thermochim. Acta, 1999, 326, 83-90

8. A.B. Buda, T. Auf der Heyde \& K. Mislow, Angew. Chem. Int. Ed., 1992, 104, 1012-1031

9. N. Weinberg \& K. Mislow, J. Math Chem. 1995, 17, 35-53 
